\title{
FATORES QUE INFLUENCIAM A FAUNA DE FORMIGAS QUE FORRAGEIA SOBRE Bauhinia monandra KURZ. (LEGUMINOSAE: CAESALPINIOIDEAE) EM ÁREA URBANA
}

\author{
FACTORS THAT AFFECT THE ANT FAUNA THAT FORAGE ON Bauhinia monandra \\ KURZ. (LEGUMINOSAE: CAESALPINIOIDEAE) IN AN URBAN AREA
}

\author{
Wésley Altino Flores ${ }^{1}$, Erika Cortines ${ }^{2}$, Ângela Alves de Almeida ${ }^{3}$, André Barbosa Vargas ${ }^{4}$, \\ Fábio Souto Almeida 5
}

\begin{abstract}
RESUMO
Os fatores que influenciam a fauna de formigas que forrageia sobre Bauhinia monandra Kurz. (Leguminosae: Caesalpinioideae) foram avaliados em área urbana no município de Três Rios, Estado do Rio de Janeiro, Brasil. As formigas foram coletadas com armadilhas arbóreas distribuídas em 15 indivíduos de $B$. monandra, realizando-se uma coleta no período de floração e outra no período sem flores. Foram obtidas 20 espécies de formigas, pertencentes a 11 gêneros e cinco subfamílias. A riqueza de espécies foi significativamente maior no período de floração (16 espécies) que no período sem flores (11 espécies). O Índice de Diversidade de Shannon também foi significativamente maior no período de floração $(2,28)$ que no período sem flores $(1,81)$. A composição da mirmecofauna variou expressivamente entre as duas épocas de coleta. O diâmetro da copa das árvores, a circunferência do tronco a altura do peito e o número de flores explicaram conjuntamente mais de $56 \%$ da variação da riqueza de espécies de formigas, por árvore. Neste sentido, a floração de $B$. monandra pode proporcionar recursos adicionais para a mirmecofauna. Além disso, os resultados demonstram que preservar as árvores mais antigas da arborização urbana e evitar podas desnecessárias podem propiciar a presença de uma maior gama de espécies de formigas sobre as árvores.
\end{abstract}

Palavras-chave: Arborização; Biodiversidade; Conservação; Formicidae; Urbanização.

\section{ABSTRACT}

The factors that affect the ant fauna that forage on Bauhinia monandra Kurz. (Leguminosae: Caesalpinioideae) were evaluated in an urban area in municipality of Três Rios, Rio de Janeiro, Brazil. The ants were collected with tree traps in 15 individuals of $B$. monandra. One collection was made in the flowering period and another in the period without flowers. We collected 20 ant species, belonging to 11 genera and five subfamilies. The species richness was significantly higher in the flowering period (16 species) that at non-flowering period (11 species). The Shannon Diversity Index was significantly higher in the flowering period (2.28) that in period without flowers (1.81). The composition of ant fauna between periods varied significantly. The crown diameter, trunk circumference and number of flowers explained together more than $56 \%$ of the variation in ant species richness per tree. The flowering of $B$. monandra provides additional resources for the ant fauna. In addition, the results showed that preserving the oldest trees in urban afforestation and avoiding unnecessary pruning can promote the presence of a wider range of ant species on trees.

Keywords: Afforestation; Biodiversity; Conservation; Formicidae; Urbanization.

Recebido em 27.11.2016 e aceito em 04.07.2017

1 Bacharel em Gestão Ambiental. Instituto Três Rios, UFRRJ. Três Rios/RJ. Email: wesleygatvc@yahoo.com.br

2 Bióloga. Doutor em Ciências Ambientais e Florestais. Professor do Departamento de Ciências do Meio Ambiente, ITR, UFRRJ. Três Rios/RJ. Email:ecortines@gmail.com

3 Graduação em Agronomia. Doutora em Entomologia. Professora do Departamento de Ciências do Meio Ambiente, ITR, UFRRJ. Três Rios/RJ. Email: aaamoth@gmail.com

4 Graduação em Ciências Biológicas. Doutor em Ciências Ambientais e Florestais. Professor do Centro Universitário de Volta Redonda (UniFOA).Volta Redonda/RJ. Email: andrebvargas@yahoo.com.br

5 Engenheiro Florestal, Doutor em Ciências Ambientais e Florestais. Professor do Departamento de Ciências do Meio Ambiente, ITR, UFRRJ. Três Rios/RJ. Email: fbio_almeida@yahoo.com.br 


\section{INTRODUÇÃO}

As formigas (Ordem Hymenoptera: Família Formicidae) são insetos abundantes, estando entre os animais mais comuns na maior parte dos ambientes terrestres do planeta (HÖLLDOBLER; WILSON, 1990). O grupo apresenta elevada riqueza taxonômica, com estimativas superiores a 20.000 espécies vivas (HÖLLDOBLER; WILSON, 1990), e uma notável diversidade funcional (GROC et al., 2014). Esta variabilidade de espécies e a facilidade para serem separadas em morfoespécies propiciam a sua utilização como organismos modelo em estudos ecológicos (RIBAS et al., 2012).

Dentre a plasticidade comportamental da família Formicidae destacam-se as interações com diversas espécies de plantas tropicais (DÁTTILO et al., 2009). Existem espécies de formigas que atuam como polinizadoras (IBARRA-ISASSI; SENDOYA, 2016). Além disso, esses insetos podem cortar folhas, dispersar sementes, forragear sobre as plantas a procura de presas e se alimentar de nectários florais e extraflorais e do honeydew produzido por hemípteros (PRINGLE et al., 2014; BACCARO et al., 2015). Outras espécies de formigas nidificam sobre as plantas e podem até mesmo protegê-las de herbívoros (DÁTTILO et al., 2009). Entretanto, a despeito dessa ampla gama de interações entre formigas e plantas, existem poucos estudos que buscaram avaliar essa relação nas áreas urbanas e conhecer seus efeitos sobre a fauna de formigas. Dentre os pontos que podem ser abordados nessas pesquisas está a influência de variações na disponibilidade de recursos ofertados pelas árvores sobre a mirmecofauna.

A fauna de formigas é abundante e diversificada mesmo em áreas urbanas (CORIOLANO et al., 2014; ESTRADA et al., 2014), e podem causar problemas, incluindo prejuízos econômicos e à saúde humana (IOP et al., 2009). Por outro lado, estudos vêm indicando a possibilidade de conservar parte da diversidade biológica do planeta em meio às áreas antropizadas e alguns já constataram que a presença das árvores é essencial para a manutenção da fauna urbana (BRUN; LINK; BRUN, 2007; CORIOLANO et al., 2014; ESTRADA et al., 2014). A arborização urbana apresenta potencial como fonte de recursos alimentares e/ou de abrigo para as formigas e diversas espécies são encontradas forrageando sobre essas plantas (DIEHL; GOTTERT; FLORES, 2006; ESTRADA et al., 2014). As comunidades de formigas podem ser utilizadas como indicadoras de diversidade biológica (RIBAS et al., 2012) e estudos sobre as relações entre formigas e plantas em áreas urbanas podem indicar formas de potencializar a conservação da biodiversidade nesses ambientes (CORIOLANO et al., 2014; ESTRADA et al., 2014).

Em regiões tropicais existem cerca de 300 espécies do gênero Bauhinia (Leguminosae: Caesalpinioideae), conhecidas como pata-de-vaca, que possuem porte arbustivo ou arbóreo e 
são comumente utilizadas na arborização urbana (LORENZI et al., 2003). Nativa do sudeste da Ásia, Bauhinia monandra Kurz pode atingir até $15 \mathrm{~m}$ de altura e $0,5 \mathrm{~m}$ de diâmetro, florescendo no inverno e no início da primavera (LORENZI et al., 2003). As folhas de B. monandra são simples, coriáceas, orbiculares e apresentam um recorte em "v" que forma dois lobos (LORENZI et al., 2003). Quanto à morfologia de sua flor, B. monandra recebe este nome por possuir apenas um estame fértil, além disso, apresenta cinco pétalas, sendo a pétala central diferenciada por apresentar uma coloração mais acentuada quando comparada as demais (QUEIROZ, 2016).

Em função da importância das formigas e por $B$. monandra ser uma espécie amplamente utilizada na arborização urbana, o presente estudo teve o objetivo de investigar os fatores que influenciam a fauna de formigas a ela associada em área urbana.

\section{MATERIAL E MÉTODOS}

O estudo foi conduzido em uma área urbana do bairro Centro do Município de Três Rios (2207'6" S; 4312'34" O), que está situado na Mesorregião Centro-Sul Fluminense, Estado do Rio de Janeiro. A arborização urbana do bairro é formada por uma considerável variedade de espécies e apresenta aproximadamente 0,6 árvores em uma extensão de $10 \mathrm{~m}$ de calçada (FARIA et al., 2013). O município apresenta uma população de cerca de 77.400 habitantes e uma área total de 326,14 km² (IBGE, 2016). A densidade demográfica do município é de 237,6 habitantes $/ \mathrm{km}^{2}$ (IBGE, 2016). O município se encontra no Bioma Mata Atlântica, com a Floresta Estacional Semidecidual (fitofisionomia natural do município) ocupando $27 \%$ do seu território (SILVÉRIO NETO et al., 2015). O clima da região é o mesotérmico, por apresentar considerável amplitude térmica (mínima de $14,4^{\circ} \mathrm{C}$ e máxima de $37,4^{\circ} \mathrm{C}$ ), além de $1.300 \mathrm{~mm}$ de chuva por ano, concentradas no verão (GOMES et al., 2013).

Foram realizadas duas coletas, diretamente em 15 árvores da espécie $B$. monandra, sendo uma coleta no mês de outubro de 2015 (quando as árvores possuíam flores) e outra em março de 2016 (sem flores). As árvores utilizadas para a coleta da mirmecofauna, no período com flores, foram as mesmas usadas no período sem flores. Para a coleta das formigas foram utilizadas armadilhas arbóreas para formigas, baseadas na proposta de Oliveira-Santos, Loyola e Vargas (2009). As armadilhas foram feitas a partir de fundos de garrafas pet com $10 \mathrm{~cm}$ de diâmetro e cortadas com $10 \mathrm{~cm}$ de altura em relação ao fundo da garrafa. No centro dos recipientes foram fixados copos descartáveis de polietileno de $20 \mathrm{~mL}$ com $4 \mathrm{~cm}$ de altura e 5 cm de diâmetro, que serviram como local para alocar a isca (sardinha em óleo comestível e mel) para atrair as formigas. A armadilha possuía ainda uma corda para facilitar a sua fixação na árvore. 
As armadilhas foram alocadas sobre as árvores a uma altura aproximada de 2,30 $\mathrm{m}$ em relação ao chão. No entorno da isca foi adicionado uma solução de $50 \mathrm{~mL}$ de água e $20 \mathrm{~mL}$ de detergente neutro para que, quando atraídas para o centro da armadilha pela isca, as formigas ficassem presas nessa solução. As armadilhas permaneceram dispostas nas árvores por um período de 48 horas. Após esse tempo, as formigas coletadas foram colocadas em recipientes com álcool $70 \%$ e transportadas para o laboratório, onde ocorreu o procedimento de triagem das amostras com a utilização de um microscópio estereoscópico. As formigas foram fixadas em via seca e posteriormente identificadas ao nível de gênero com base em Baccaro et al. (2015). A identificação ao nível de espécie teve como base revisões taxonômicas específicas para os gêneros amostrados e também comparações com espécimes previamente identificados.

A temperatura do ar foi obtida com um termômetro alocado próximo de cada árvore avaliada. Também foram coletadas, de cada árvore, a circunferência do tronco à altura do peito (CAP) e o diâmetro da copa, nos sentidos transversal e paralelo às calçadas com ajuda de uma trena (ver CORIOLANO et al., 2014). Além disso, a copa de $B$. monandra foi dividida visualmente em quatro quadrantes e obteve-se uma estimativa do número de flores em um dos quadrantes.

Para a análise dos dados, foi utilizado o Teste de Qui-Quadrado para verificar diferenças nas frequências das espécies de formigas mais abundantes nas épocas com e sem flores. Para avaliar a riqueza total de formigas, curvas de acumulação de espécies foram obtidas para as duas épocas de coleta. $\mathrm{Na}$ análise da riqueza média por armadilha foi utilizado o teste t pareado. Foi calculado o Índice de Diversidade de Shannon e realizado o teste t para esse índice (Diversity $t$ test), no programa PAST (HAMMER; HARPER; RYAN, 2001). Para analisar a composição de espécies de formigas foi realizada uma Análise de Componentes Principais (PCA). Por fim, para verificar a influência do diâmetro da copa, da circunferência do tronco e do número de flores sobre a riqueza de espécies de formigas foi utilizada a regressão múltipla passo-a-passo.

\section{RESULTADOS E DISCUSSÃO}

Foram obtidas 20 espécies de formigas, pertencentes a 11 gêneros e cinco subfamílias (Tabela 1). Foram encontradas oito espécies da subfamília Formicinae, seis da Myrmicinae, três da Pseudomyrmecinae, duas da Dolichoderinae e uma da Ponerinae. As copas das árvores podem ser consideradas como habitats mais hostis para as formigas que a interface solo-serapilheira, pois possuem geralmente menos umidade, são mais expostas às intempéries climáticas e possuem menos locais para nidificação (HÖLLDOBLER; WILSON, 1990). Contudo, 
várias espécies de formigas têm adaptações que as permitem nidificar e forragear nas copas das árvores, observando-se, como no presente estudo, uma vasta diversidade de espécies de formigas ocupando esse habitat.

Tabela 1. Subfamílias e espécies de formigas coletadas sobre Bauhinia monandra Kurz. (Leguminosae: Caesalpinoideae) no período com e sem floração, no município de Três Rios, Estado do Rio de Janeiro, Brasil

Table 1. Subfamilies and ant species collected on Bauhinia monandra Kurz. (Leguminosae: Caesalpinoideae) in the period with and without flowers, in the municipality of Três Rios, Rio de Janeiro State, Brazil

\begin{tabular}{|c|c|c|}
\hline Subfamílias/Espécies & Com Floração & Sem Floração \\
\hline \multicolumn{3}{|l|}{ Dolichoderinae } \\
\hline Dorymyrmex sp.1 & - & 3 \\
\hline Tapinoma melanocephalum Fabricius, 1793 & 1 & 1 \\
\hline \multicolumn{3}{|l|}{ Formicinae } \\
\hline Brachymyrmex sp. 1 & 6 & 2 \\
\hline Camponotus fastigatus Roger, 1863 & 1 & 1 \\
\hline Camponotus sp.1 & 5 & 2 \\
\hline Camponotus sp.3 & 1 & - \\
\hline Camponotus sp.4 & 1 & - \\
\hline Camponotus sp.5 & - & 2 \\
\hline Camponotus sp.6 & - & 1 \\
\hline Paratrechina longicornis Latreille, 1802 & 2 & 10 \\
\hline \multicolumn{3}{|l|}{ Myrmicinae } \\
\hline Cephalotes pusillus (Klug, 1824) & 1 & - \\
\hline Monomorium floricola (Jerdon, 1851) & 2 & 1 \\
\hline Pheidole prox. fallax Mayr, 1870 & 1 & - \\
\hline Pheidole sp.1 & 3 & 10 \\
\hline Solenopsis sp. 1 & 1 & - \\
\hline Solenopsis sp.2 & 1 & - \\
\hline \multicolumn{3}{|l|}{ Ponerinae } \\
\hline Odontomachus sp.1 & 1 & - \\
\hline \multicolumn{3}{|l|}{ Pseudomyrmecinae } \\
\hline Pseudomyrmex sp.1 & 2 & - \\
\hline Pseudomyrmex sp.2 & 1 & - \\
\hline Pseudomyrmex sp.3 & - & 1 \\
\hline Riqueza de Espécies & 16 & 11 \\
\hline Índice de Diversidade de Shannon & 2,28 & 1,81 \\
\hline
\end{tabular}

Camponotus foi o gênero mais rico em espécies, seguido por Pseudomyrmex. Vários estudos constataram a elevada riqueza de espécies de Camponotus em áreas urbanas e evidenciaram a ampla presença de espécies desse gênero sobre a arborização urbana (CORIOLANO et al., 2014; ESTRADA et al., 2014). Este gênero também apresentou expressividade $(40,9 \%$ da riqueza total) em comunidades de formigas que forrageiam sobre as espécies utilizadas na arborização de São Leopoldo-RS (DIEHL; GOTTERT; FLORES, 2006). Algumas espécies de Camponotus são consideradas como pragas e nidificam em cavidades 
no tronco ou galhos de árvores, podendo causar danos às plantas quando escavam tais cavidades (GONÇALVES; LOECK; ROSADO, 2014; HÖLLDOBLER; WILSON, 1990).

O gênero Pseudomyrmex é composto, quase que totalmente, por espécies arborícolas (BACCARO et al., 2015) e é comumente encontrado em áreas urbanas no Brasil (DIEHL; GOTTERT; FLORES, 2006; ESTRADA et al., 2014). Cabe ressaltar o baixo número de espécies do gênero Pheidole, que frequentemente é o mais rico em espécies em ambientes naturais da região Neotropical (HÖLLDOBLER; WILSON, 1990).

No período da floração foram coletados 10 gêneros, enquanto que na época sem floração foram oito gêneros. Os gêneros Cephalotes e Odontomachus ocorreram apenas na época com floração, representados por apenas uma espécie cada, que foi amostrada em apenas uma árvore. Cephalotes é um gênero composto por muitas espécies arborícolas, cujos indivíduos podem se alimentar em nectários (MELO et al., 2010). O gênero Odontomachus apresenta espécies que são tipicamente predadoras, mas que também consomem vários outros tipos de alimentos, incluindo exsudados de plantas (ALMEIDA; MAYHE-NUNES; QUEIROZ, 2013; BACCARO et al., 2015). Devido a sua baixa frequência, não se pode afirmar que a presença dessas formigas sobre as árvores tenha relação com os recursos propiciados pela floração. Do mesmo modo, não é possível afirmar que a espécie do gênero Dorymyrmex, encontrada apenas na época sem floração, não utilize os recursos alimentares das flores de $B$. monandra.

As espécies mais frequentes na época da floração foram Brachymyrmex sp.1 e Camponotus sp.1, contudo a frequência de Brachymyrmex sp.1 $\left(x^{2}=2,00 ; p=0,16\right)$ e de Camponotus sp. $1\left(X^{2}=1,29 ; p=0,26\right)$ não diferiram significativamente entre as épocas com e sem floração. Por outro lado, a frequência de Paratrechina longicornis Latreille $\left(x^{2}=5,333 ; p=\right.$ $0,02)$ e de Pheidole sp.1 ( $\left.x^{2}=3,77 ; p=0,05\right)$ foram significativamente maiores na época sem floração. Assim, as diferenças observadas podem indicar uma possível competição entre as espécies de formigas. A maior presença, de outras espécies de formigas no período de floração, pode estar contribuindo para a redução da frequência de $P$. longicornis e Pheidole sp.1 na época que as árvores apresentam flores. Dentre as espécies amostradas, cabe destacar que $P$. longicornis e Tapinoma melanocephalum (Fabricius) estão entre as principais espécies de formigas consideradas pragas nas áreas urbanas de várias regiões brasileiras (IOP et al., 2009; CORIOLANO et al., 2014).

Pela curva de acumulação de espécies pode-se observar que a riqueza total de espécies formigas foi significativamente maior no período de floração comparado ao período sem floração (Figura 1). Por outro lado, a riqueza média por árvore não diferiu significativamente entre as épocas de amostragem (Teste t pareado; $t=0,81 ; p=0,43$; Figura 
2). O Índice de Diversidade de Shannon também foi significativamente maior no período com floração que no período sem floração (Diversity t test, $\mathrm{t}=2,01 ; \mathrm{p}=0,048$; Tabela 1).

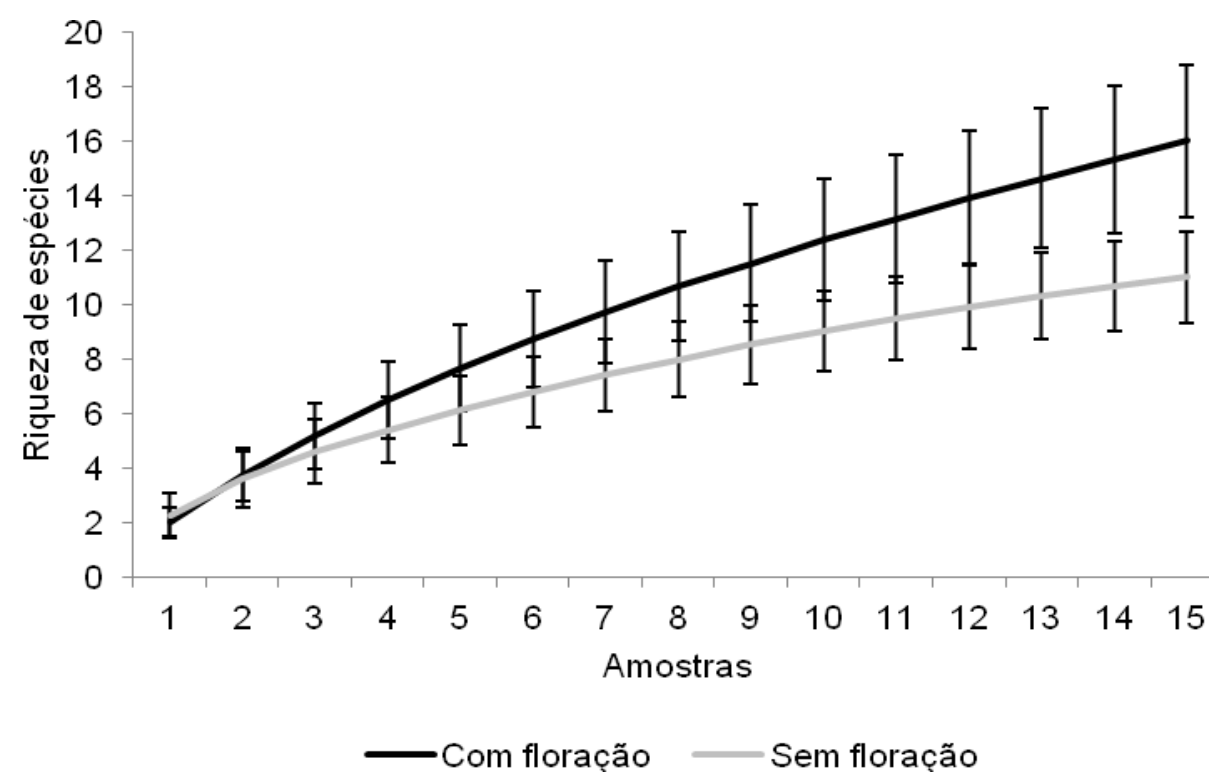

Figura 1. Curva de acumulação de espécies de formigas sobre Bauhinia monandra Kurz (Leguminosae: Caesalpinoideae), nos períodos com e sem floração, em área urbana do município de Três Rios, Rio de Janeiro, Brasil.

Figure 1. Species accumulation curve for ant fauna on Bauhinia monandra Kurz (Leguminosae: Caesalpinoideae), in the periods with and without flowers, in an urban area of the municipality of Três Rios, Rio de Janeiro State, Brazil

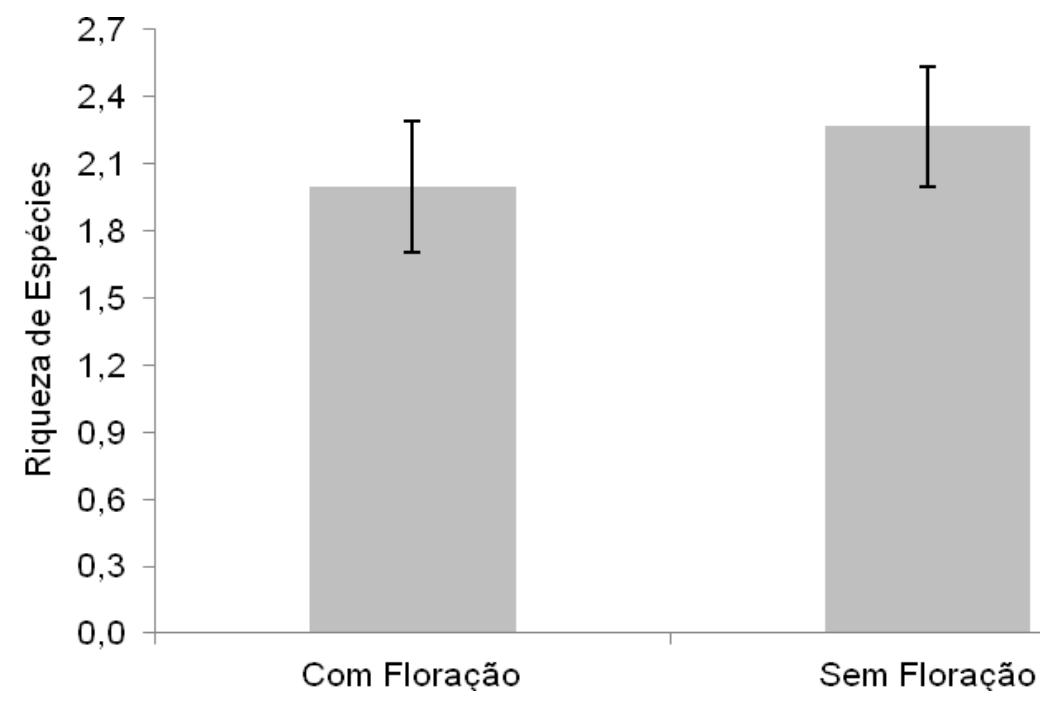

Figura 2. Riqueza média de espécies de formigas por armadilha sobre Bauhinia monandra Kurz (Leguminosae: Caesalpinoideae), nos períodos com e sem floração, em área urbana do município de Três Rios, Rio de Janeiro, Brasil.

Figure 2. Average ant species richness by trap on Bauhinia monandra Kurz (Leguminosae: Caesalpinoideae), in the periods with and without flowers, in an urban area of the municipality of Três Rios, Rio de Janeiro State, Brazil. 
Vale ressaltar que a temperatura do ar influencia a reprodução e as atividades dos insetos e, geralmente, as temperaturas maiores são mais propícias aos mesmos, inclusive para as formigas (ALMEIDA; QUEIROZ; MAYHÉ-NUNES, 2007). É esperado que no final do verão ocorram populações com maior número de formigas operárias e que essas estejam mais ativas, sendo mais facilmente capturadas em armadilhas de queda. Porém, a maior diversidade de formigas foi encontrada na primavera, em dias que a temperatura do ar estava na faixa de $25,3^{\circ} \mathrm{C}$ (época de floração da $B$. monandra), e não no final do verão, quando a temperatura do ar média nos dias de coleta foi de $31,5^{\circ} \mathrm{C}$. Isso fortalece a hipótese de que a presença das flores em $B$. monandra seja o fator que proporcionou o aumento da riqueza de espécies de formigas.

A análise de componentes principais indicou variação na composição da fauna de formigas entre os períodos com e sem floração (Figura 3). Assim, existe uma variação expressiva das espécies que forrageiam sobre $B$. monandra no período em que as árvores apresentam flores, comparado ao período em que não possuem flores.

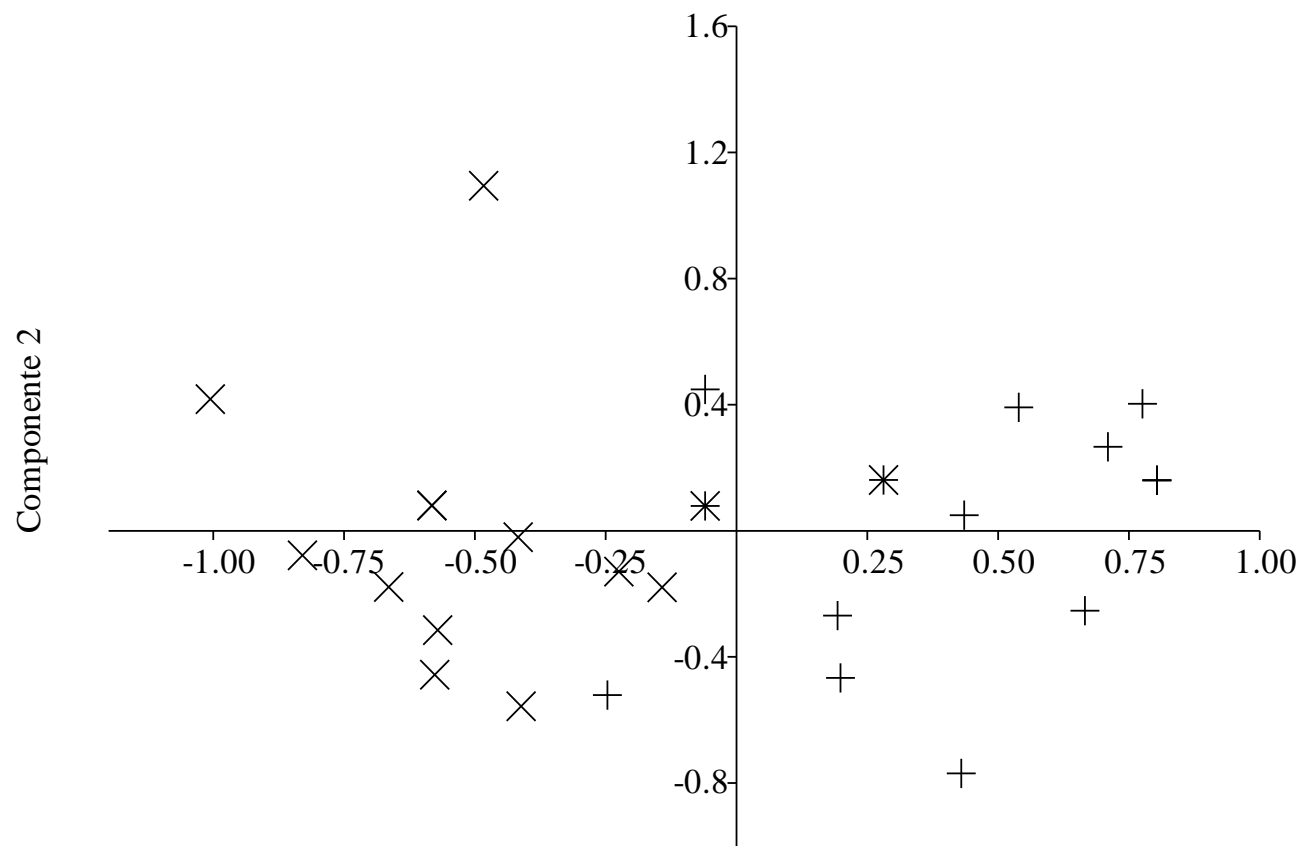

Componente 1

Figura 3. Análise de Componentes Principais (PCA) para a fauna de formigas coletada sobre árvores da espécie Bauhinia monandra Kurz. (Leguminosae: Caesalpinoideae) com floração $(x)$ e sem floração (+), no município de Três Rios, Rio de Janeiro, Brasil.

Figure 3. Principal Component Analysis (PCA) for the ant fauna collected on Bauhinia monandra Kurz. (Leguminosae: Caesalpinoideae), with flowering ( $\mathrm{x}$ ) and without flowering $(+)$, in the municipality of Três Rios, Rio de Janeiro State, Brazil. 
O diâmetro da copa foi a característica das árvores que mais afetou a riqueza de espécies de formigas por árvore (Tabela 2). Essa variável explicou mais de 52\% da variação da riqueza de espécies. Ao se inserir as outras duas variáveis independentes (circunferência do tronco e número de flores), o modelo matemático explicou significativamente cerca de $56 \%$ da variação da riqueza de espécies de formigas por árvore de $B$. monandra.

Tabela 2. Regressão múltipla passo-a-passo com a riqueza de espécies de formigas por árvore (variável dependente) da espécie Bauhinia monandra Kurz. (Leguminosae: Caesalpinoideae) e as variáveis independentes (diâmetro da copa - DC, circunferência do tronco à altura do peito CAP e número de flores - NF) no município de Três Rios, Rio de Janeiro, Brasil

Table 2. Stepwise regression with the ant species richness by tree (dependent variable) of the species Bauhinia monandra Kurz. (Leguminosae: Caesalpinoideae) and the independent variables (crown diameter - DC, trunk circumference - CAP and number of flowers - NF) in the municipality of Três Rios, Rio de Janeiro State, Brasil

\begin{tabular}{ccccc}
\hline Var. Dependente (Y): Riqueza & $\mathrm{R}^{2}$ & Variação R & $\mathrm{F}$ & $\mathrm{P}$ \\
\hline DC & $52,36 \%$ & $52,36 \%$ & 14,290 & $<0,01$ \\
DC, CAP & $53,32 \%$ & $0,96 \%$ & 6,855 & 0,01 \\
DC, CAP, NF & $56,25 \%$ & $2,93 \%$ & 4,715 & 0,02 \\
\hline
\end{tabular}

Nota-se que os indivíduos de $B$. monandra de maior porte possuem maior riqueza de espécies de formigas. Assim, no que tange a proteção da fauna nas áreas urbanas, que é maximizada com a presença de árvores (BRUN; LINK; BRUN, 2007; ESTRADA et al., 2014), a existência de árvores de grande porte pode trazer benefícios, devendo os gestores públicos evitar o corte desses indivíduos e podas desnecessárias.

\section{CONCLUSÕES}

A partir dos resultados observados pode-se inferir que a floração de $B$. monandra propicia recursos adicionais para a fauna de formigas em área urbana. A composição de espécies da mirmecofauna variou nos períodos com e sem floração. Em árvores de maior porte foi registrada maior riqueza de espécies de formigas.

\section{REFERÊNCIAS}

ALMEIDA, F.S.; MAYHE-NUNES, A.J.; QUEIROZ, J.M. The importance of poneromorph ants for seed dispersal in altered environments. Sociobiology, Feira de Santana, v.60, n.3, p.229235, 2013.

ALMEIDA, F.S.; QUEIROZ, J.M.; MAYHÉ-NUNES, A. Distribuição e abundância de ninhos de Solenopsis invicta Buren (Hymenoptera: Formicidae) em um agroecossistema diversificado sob manejo orgânico. Floresta e Ambiente, Seropédica, v.14, n.1, p.33-43, 2007. 
BACCARO, F.B.; FEITOSA, R.M.; FERNANDES, F.; FERNANDES, I.O.; IZZO, T.J.; SOUZA, J.L.P.; SOLAR, R. Guia para os gêneros de formigas do Brasil. Manaus: INPA, 2015. 388p.

BRUN, F.G.K.; LINK, D.; BRUN, E.J. O emprego da arborização na manutenção da biodiversidade de fauna em área urbanas. Revista da Sociedade Brasileira de Arborização Urbana, Piracicaba, v.2, n.1, p.117-127, 2007.

CORIOLANO, R.E.; ESTRADA, M.A.; SANTOS, N.T.; CAIXEIRO, L.R.; VARGAS, A.B.; ALMEIDA, F.S. Mirmecofauna associada à arborização urbana no município de Três Rios, RJ, Brasil. Revista Brasileira de Biociências, Porto Alegre, v.12, n.4, p.210-214, 2014.

DÁTTILO, W.; MARQUES, E.C.; FALCÃO, J.C.F.; MOREIRA, D.D.O. Interações mutualísticas entre formigas e plantas. Entomobrasilis, Vassouras, v.2, n.2, p.32-36, 2009.

DIEHL, E.; GOTTERT, C.L.; FLORES, D.G. Comunidades de formigas em três espécies utilizadas na arborização urbana em São Leopoldo, Rio Grande do Sul, Brasil. Bioikos, Campinas, v.20, n.1, p.25-32, 2006.

ESTRADA, M.A.; CORIOLANO, R.E.; SANTOS, N.T.; CAIXEIRO, L.R.; VARGAS, A.B.; ALMEIDA, F.S. Influência de áreas verdes urbanas sobre a mirmecofauna. Floresta e Ambiente, Seropédica, v.21, n.2, p.162-169, 2014.

FARIA, D.C.; DUARTE, J.M.A.; PINTO, D.M.; ALMEIDA, F.S. Arborização urbana no município de Três Rios-RJ: espécies utilizadas e a percepção de seus benefícios pela população. Revista da Sociedade Brasileira de Arborização Urbana, Piracicaba, v.8, n.2, p.58-67, 2013.

GOMES, O.V.O.; MARQUES, E.D.; SOUZA, M.D.C.; SILVA FILHO, E.V. Influência antrópica nas águas superficiais da cidade de Três Rios, (RJ). Geochimica Brasiliensis, Recife, v.27, p.79-88, 2013.

GONÇALVES, M.G.; LOECK, A.E.; ROSADO, J.L.O. Primeiro registro de Camponotus cingulatus Mayr, 1862 (Hymenoptera: Formicidae) para o estado do Rio Grande do Sul, Brasil. Arquivos do Instituto Biológico, São Paulo, v.81, n.1, p.68-70, 2014.

GROC, S.; DELABIE, J.H.C.; FERNANDEZ, F.; LEPONCE, M.; ORIVEL, J.; SILVESTRE, R.; VASCONCELOS, H.L.; DEJEAN, A. Leaf-litter ant communities (Hymenoptera: Formicidae) in a pristine Guianese rain-forest: stable functional structure versus high species turnover. Myrmecological News, Viena, v.19, p.43-51, 2014.

HAMMER, R.; HARPER, D.A.T.; RYAN, P.D. PAST: Paleontological statistics software package for education and data analysis. Palaeontologia Electronica, California, v.4, n.1, 9p., 2001.

HÖLLDOBLER, B.; WILSON, E.O. The ants. 1ed. Cambridge: Harvard University Press. 1990. $733 p$.

IBARRA-ISASSI, J.; SENDOYA, S.F. Ants as floral visitors of Blutaparon portulacoides (A. StHil.) Mears (Amaranthaceae): an ant pollination system in the Atlantic Rainforest. ArthropodPlant Interactions, New York, v.10, n.3, p.221-227, 2016.

IBGE - Instituto Brasileiro de Geografia e Estatística. Disponível em: $<$ http://cidades.ibge.gov.br/painel/painel.php?codmun=330600>. Acesso em: 15 fev. 2016.

IOP, S.; CALDART, V.M.; LUTINSKI JUNIOR, A.; GARCIA, F.R.M. Formigas urbanas da cidade de Xanxerê, Santa Catarina, Brasil. Biotemas, Florianópolis, v.22, n.2, p.55-64, 2009. 
LORENZI, H.; SOUZA, H.M.; TORRES, M.A.V.; BACHER, L.B. Árvores exóticas no Brasil: madeireiras, ornamentais e aromáticas. Nova Odessa: Instituto Plantarum, 2003. 368p.

MELO, Y.; CÓRDULA, E.; MACHADO, S.R.; ALVES, M. Morfologia de nectários em Leguminosae senso lato em áreas de caatinga no Brasil. Acta Botanica Brasilica, Belo Horizonte, v.24, n.4., p.1034-1045, 2010.

OLIVEIRA-SANTOS, L.G.R.; LOYOLA, R.D.; VARGAS, A.B. Armadilhas de dossel: uma técnica para amostrar formigas no estrato vertical de florestas. Neotropical Entomology, Londrina, v.38, n.5, p.691-694, 2009.

PRINGLE, E.G.; NOVO, A.; ABLESON, I.; BARBEHENN, R.V.; VANNETTE, R.L. Plant-derived differences in the composition of aphid honeydew and their effects on colonies of aphid-tending ants. Ecology and Evolution, Malden, v.4, n.21, p.4065-4079, 2014.

QUEIROZ RT. Disponível em: <http://rubens-plantasdobrasil.blogspot.com.br/2014/09/ fabaceae-bauhinia-monandra-kurz.html>. Acesso em: 15 mai. 2016.

RIBAS, C.R.; CAMPOS, R.B.F.; SCHMIDT, F.A.; SOLAR, R.R.C. Ants as indicators in Brazil: a review with suggestions to improve the use of ants in environmental monitoring programs. Psyche, New York, ID636749, 23p., 2012

SILVÉRIO NETO, R.; BENTO, M.C.; MENEZES, S.J.M.C.; ALMEIDA, F.S. Caracterização da cobertura florestal de unidades de conservação da Mata Atlântica. Floresta e Ambiente, Seropédica, v.22, n.1, p.32-41, 2015. 